Épijournal de Géométrie Algébrique

epiga.episciences.org

Volume 1 (2017), Article Nr. 1

EPIGA

\title{
Conic bundles that are not birational to numerical Calabi-Yau pairs
}

\author{
János Kollár
}

\begin{abstract}
Let $X$ be a general conic bundle over $\mathbb{P}^{2}$ with branch curve of degree at least 19 . We prove that there is no normal projective variety $Y$ that is birational to $X$ and such that some multiple of its anticanonical divisor is effective.
\end{abstract}

Keywords. Rationally connected variety, conic bundle, Calabi-Yau variety, birational equivalence.

2010 Mathematics Subject Classification. 14M22, 14J45, 14J20 (Primary); 14J32, $14 \mathrm{E} 05$ (Secondary).

\section{[Français]}

Titre. Fibrés en coniques qui ne sont pas birationnels à des paires de Calabi-Yau numériques

Résumé. Soit $X$ un fibré en coniques général sur $\mathbb{P}^{2}$ avec une courbe de branchement de degré au moins 19. Nous montrons qu'il n'existe pas de variété projective normale $Y$ qui soit birationnelle à $X$ et telle qu'un multiple de son diviseur anticanonique soit effectif.

Received by the Editors on June 24, 2016, and in final form on January 17, 2017.

Accepted on January 30, 2017.

János Kollár

Princeton University, Princeton NJ 08544-1000

e-mail: kollar@math.princeton.edu

Partial financial support was provided by the NSF under grant number DMS-1362960.

(C) by the author(s)

This work is licensed under http://creativecommons.org/licenses/by-sa/4.0/ 
Understanding the difference between rationally connected varieties and Fano varieties has long been a goal of birational geometry. In any dimension, smooth Fano varieties come in finitely many families but rationally connected varieties form infinitely many families. Thus one expects that not every rationally connected variety is birational to a Fano variety, but actual proofs of this fact have been quite subtle; see [Sar80] or [Cor95].

A more general form of this problem asks if every rationally connected variety $X$ is birational to the underlying variety of a $\mathbb{Q}$-Fano pair $(Y, \Delta)$. Since $\mathbb{Q}$-Fano pairs form infinitely many families up-to birational equivalence [Oka09], this is a harder variant. This form of the question was posed in [CG13] and a negative answer is established in [Kry14]. Closely related results describing Calabi-Yau fiber-space structures on certain Fano 3-folds are proved in [Che04, Ryd06, CK10].

Probably the most general question in this direction is whether every rationally connected variety is birational to the underlying variety of a numerical Calabi-Yau pair $(Y, \Delta)$. Here we allow the most general definition of Calabi-Yau pairs, that is, $Y$ is normal, proper, $\Delta$ is a pseudo-effective $\mathbb{R}$-divisor, $K_{Y}+\Delta$ is $\mathbb{R}$-Cartier and $K_{Y}+\Delta \equiv 0$, but we impose no restrictions on the singularities of $Y$. (For most purposes the more restrictive definition of Calabi-Yau pairs adopted in [KX16] is the best; but the above numerical version also seems natural.) It turns out that the singularities of $Y$ are not very important and the difference between effective and pseudo-effective divisors also may not be significant, but allowing divisors with coefficient $>1$ in $\Delta$ leads to many more cases; see Example 4 and Definition 6. If $X$ is birational to a numerical Calabi-Yau pair $(Y, \Delta)$ then the birational transform of $\Delta$ on $X$ is frequently a quite interesting divisor. Understanding such divisors was a key to proving unirationality of degree 1 conic bundle surfaces [KM17].

We discuss several methods to show that certain conic bundles (see Definition 5) are not birational to any numerical Calabi-Yau pair. Typical results are the following.

Theorem 1. There are conic bundles $S \rightarrow \mathbb{P}^{1}$ defined over $\mathbb{Q}$ that are not birational to any numerical Calabi-Yau surface.

This property seems to depend very subtly on the coefficients involved in the definition of $S$ and we give only sufficient conditions in Example 23. The following are some concrete special cases.

Example 2. Let $p \geq 11$ be a prime such that $p \equiv-1 \bmod 4$ and choose $m \in \mathbb{Z}$ not divisible by $p$. Then the conic bundle

$$
\left(z^{2}=\left(\frac{s^{p-1}-1}{s^{2}-1}\right) x^{2}+\left(s^{p-1}-1+m p\right) y^{2}\right) \subset \mathbb{P}_{x y z}^{2} \times \mathbb{A}_{s}^{1}
$$

is not birational—over $\mathbb{Q}$ - to any numerical Calabi-Yau surface.

In dimension 3 we get the following.

Theorem 3. Let $X_{d, 2} \subset \mathbb{P}^{2} \times \mathbb{P}^{2}$ be a general hypersurface of bidegree $(d, 2)$ over a field of characteristic $\neq 2,3,5$. Then $X_{d, 2}$ is not birational to the underlying variety of a numerical Calabi-Yau pair for $d \geq 7$.

I could not write down explicit examples with smooth branch curve, but it is easy to get many with reducible branch curve using Corollary 17.

It is quite likely that the theorem also holds over any infinite field. The main arguments in our paper work whenever the characteristic is $\neq 2$, but a key reference [Sar80] is stated in the literature only for characteristic $\neq 2,3$ and we also use resolution of singularities.

The most important numerical invariant of a conic bundle $\pi: X \rightarrow \mathbb{P}^{n}$ is the degree of its branch divisor $B_{X} \subset \mathbb{P}^{n}$. Typical results say that for smooth branch divisors the degree is the only important invariant and the higher the degree of $B_{X}$, the more complicated $X$ is. This is, however, not the case 
for our question. We see in Examples 20-21 that there are conic bundles $\pi: X \rightarrow \mathbb{P}^{2}$ with smooth branch curve of arbitrary high degree (resp. $\pi: S \rightarrow \mathbb{P}^{1}$ with many singular fibers) that are birational to numerical Calabi-Yau varieties.

The following example shows that many varieties are birational to the underlying variety of a smooth numerical Calabi-Yau pair.

Example 4. If $(Y, \Delta)$ is a numerical Calabi-Yau pair and $\Delta \neq 0$ then $Y$ is uniruled by [MM86] but $Y$ need not be rationally connected. In fact, for any smooth, projective variety $X$, the product $X \times \mathbb{P}^{1}$ is birational to a numerical Calabi-Yau pair. To see this, let $H$ be an ample divisor on $X$ such that $H-K_{X}$ is effective and note that the anticanonical class of $\mathbb{P}_{X}\left(\mathcal{O}_{X} \oplus \mathcal{O}_{X}(H)\right)$ is effective. It is the sum of twice the negative section and of the pull-back of $H-K_{X}$.

In particular, the conic bundles in Theorem 3 are not birationally ruled. However, we use even stronger non-rationality results of [Sar80] during the proof.

Definition 5. A conic bundle is a flat, proper morphism $\pi: X \rightarrow Z$ such that every fiber of $\pi$ is isomorphic to a plane conic. We are mainly interested in the cases when $Z$ is regular, but for the basic definition it is enough to assume that $Z$ is normal. (For many purposes one should allow non-flat morphisms and more singular fibers, but for us the restrictive version is more convenient.)

A conic bundle is called extremal if for every codimension 1 point $z \in Z$ the fiber $X_{z}$ is irreducible over $k(z)$. Equivalently, the relative Picard number is 1.

A conic bundle is called minimal if it is extremal and has no rational sections. (This is the "right" definition for conic bundles but note that a product $Z \times \mathbb{P}^{1} \rightarrow Z$ is extremal but not minimal in our sense.) If $X$ is regular then $\pi: X \rightarrow Z$ is minimal iff $\operatorname{Pic}(X)=\pi^{*} \operatorname{Pic}(Z) \oplus \mathbb{Z}\left[\omega_{X / Z}^{-1}\right]$; this is the key property that we are interested in. This is equivalent to saying that if $L$ is any line bundle on $X$ and $C \subset X$ is a fiber then $\operatorname{deg}\left(\left.L\right|_{C}\right)$ is even and if $C$ is reducible then $\left.L\right|_{C}$ has the same degree on both irreducible components.

The branch locus, denoted by $B_{X} \subset Z$, is the subscheme parametrizing singular fibers of $\pi$. Set theoretically it consists of points $z \in Z$ such that $X_{z}:=\pi^{-1}(z)$ is singular. If $X$ is regular then $B_{X}$ is reduced. In general, the scheme structure is given by the formula (25.3) which also shows that $B_{X}$ has pure codimension 1 in $Z$, except possibly when char $k(Z)=2$. In order to avoid various complications, we assume from now on that all residue characteristics are $\neq 2$. Our main interest is in conic bundles over $\mathbb{C}$ or $\mathbb{Q}$, but we will use some examples that are defined over $\mathbb{Z}_{p}$.

Assume that $Z$ is regular and let $z \in B_{X}$ be a regular point. Then $X_{z}$ is a pair of lines and $X$ is regular along $X_{z}$; see Paragraph 25. This defines a double cover $\tilde{B}_{X} \rightarrow B_{X}$ that is étale over the regular locus of $B_{X}$. Then $X \rightarrow Z$ is minimal iff, for every irreducible component $B_{i} \subset B_{X}$, the corresponding $\tilde{B}_{i}$ is also irreducible.

I do not know a good general introduction to conic bundles, but everything can be gleaned from the basic sources [Isk67, Bea77, Sar80, Isk87] or [Cor00, Chap.4], [KSC04, 3.11-13]. The key results are discussed in Paragraphs 25-28.

Definition 6. Let $k$ be a field and $X$ a normal, proper $k$-variety of dimension $n$. A divisor class $B$ is called effective (resp. $\mathbb{Q}$-effective) if $B$ is linearly (resp. $\mathbb{Q}$-linearly) equivalent to an effective $\mathbb{Z}$-divisor (resp. $\mathbb{Q}$-divisor). An $\mathbb{R}$-divisor $B$ is called pseudo-effective if its class in $N_{n-1}(X)_{\mathbb{R}}$ is a limit of $\mathbb{Q}$-effective divisors.

Birational transformation of divisors does not preserve linear equivalence, so it is not very useful to ask whether a divisor is birationally effective or not. However, the birational transform of a mobile linear system is well defined and the canonical class makes sense on any birational model of $X$.

Let $|M|$ be a mobile linear system on $X$. We say that $-K_{X}+|M|$ is birationally effective (resp. birationally $\mathbb{Q}$-effective or birationally pseudo-effective) if there is a normal, proper $k$-variety $\phi: X^{\prime} \stackrel{b i r}{\sim}$ $X$ such that $-K_{X^{\prime}}+\phi_{*}|M|$ is effective (resp. $\mathbb{Q}$-effective or pseudo-effective). 
For now our main interest is in the case $|M|=0$.

We stress that we do not impose a priori restrictions on the singularities of $X^{\prime}$ but it is easy to improve them, at least in characteristic 0 .

Assume that $-K_{X^{\prime}} \sim_{\mathbb{Q}} D^{\prime}$ is pseudo-effective and let $p: X^{\prime \prime} \rightarrow X^{\prime}$ be a terminal modification $\left[\right.$ Kol13, 1.33]. Write $-K_{X^{\prime \prime}} \sim_{\mathbb{Q}} E^{\prime \prime}+D^{\prime \prime}$ where $E^{\prime \prime}$ is $p$-exceptional and $D^{\prime \prime}$ is the birational transform of $D^{\prime}$. By the Negativity lemma $[K M 98,3.39]$ we see that $E^{\prime \prime}$ is an effective $\mathbb{R}$-divisor, hence $-K_{X^{\prime \prime}}$ is pseudo-effective.

If $K_{X^{\prime \prime}}$ is not numerically trivial, then it is also not pseudo-effective, hence a suitable minimal model program terminates with a Mori fiber space $X^{m} \rightarrow Z$. That is, $-K_{X^{m}}$ is relatively ample and the relative Picard number of $X^{m} / Z$ is 1 . Note that $\phi: X^{\prime \prime} \rightarrow X^{m}$ is a birational contraction, that is, $\phi^{-1}$ has no exceptional divisors. Thus if $-K_{X^{\prime \prime}} \sim_{\mathbb{Q}} D^{\prime \prime}$ then $-K_{X^{m}}=-\phi_{*}\left(K_{X^{\prime \prime}}\right) \sim_{\mathbb{Q}} \phi_{*}\left(D^{\prime \prime}\right)=: D^{m}$ shows that $-K_{X^{m}}$ is also pseudo-effective.

Thus the following holds.

Claim 6.1. Let $k$ be a field of characteristic 0 and $X$ a normal $k$-variety. Then $-K_{X}$ is birationally pseudo-effective iff

(a) either $X$ has canonical singularities and $K_{X}$ is numerically trivial,

(b) or there is a projective variety $X^{m}$ with terminal singularities such that $X^{m}$ is birational to $X$, $-K_{X^{m}}$ is pseudo-effective and there is a Mori fiber space structure $\pi: X^{m} \rightarrow Z$.

This suggests that our question should be treated using the Noether-Fano method. (See [KSC04, Chap.5] for an introduction and [Cor00] for a more detailed treatment.) This is the approach taken in [Kry14] and the examples given there are also not birational to a numerical Calabi-Yau pair. We work with conic bundles. These have a rich birational geometry yet all of their birational models are quite well understood.

The characteristic 0 assumption is only needed to guarantee that resolutions and Mori fiber space models exist. Thus Claim 6.1 holds over any field $k$ if $\operatorname{dim} X=2$ or if $\operatorname{dim} X=3$ and $\operatorname{char} k>5$.

Note also that in birational geometry one would usually like to control the singularities of the pair $\left(X^{\prime}, \Delta^{\prime}\right)$, not just the singularities of $X^{\prime}$. However, even if $\Delta^{\prime}$ is effective, we do not assume that the coefficients in $\Delta^{\prime}$ are $\leq 1$, thus a similar reduction to the log canonical case is not possible.

Remark 7. Our notion is close to the concept of Fano type varieties introduced in [PS09], which asks for a normal, proper $k$-variety $X^{\prime} \stackrel{b i r}{\sim} X$ such that $-K_{X^{\prime}} \sim \mathbb{Q} \Delta^{\prime}+H^{\prime}$ where $\left(X^{\prime}, \Delta^{\prime}\right)$ is klt and $H^{\prime}$ is an ample $\mathbb{Q}$-divisor. (That is, $\left(X^{\prime}, \Delta^{\prime}\right)$ is a log-Fano pair.) ¿From the technical point of view the difference between $-K_{X}$ being birationally pseudo-effective and $X$ being of Fano type can be substantial, but in our examples none of the difficulties appear.

8. (Outline of the proof of Theorems 1 and 3) Start with $X \rightarrow \mathbb{P}^{2}$ over $\mathbb{C}$ and restrict to a generic line $\mathbb{P}^{1} \subset \mathbb{P}^{2}$. We get a 2 -dimensional conic bundle $S \rightarrow \mathbb{P}^{1}$ over the function field $\mathbb{C}(s, t)$. A slight complication is that $-\left.K_{X}\right|_{S}=-K_{S}+F$ where $F$ is a general fiber, but this is easy to deal with. Next we study when $-K_{S}+F$ is birationally pseudo-effective for 2-dimensional conic bundles $S \rightarrow \mathbb{P}^{1}$ over $\mathbb{C}(s, t)$, or, more generally, over any field $k$. We show in Corollary 11 that this holds iff $S$ contains a double section $C \subset S$ whose normalization has genus $\leq 2$. The double section $C \subset S$ then extends to a generically finite double section $D \subset X$ whose normalization is a birational to a K3 surface. (The projection $\pi_{D}: D \rightarrow \mathbb{P}^{2}$ has degree 2 and the ramification curve is a sextic, but $\pi_{D}$ need not be finite.) Then we study how the branch curve of $X \rightarrow \mathbb{P}^{2}$ and the branch curve of $D \rightarrow \mathbb{P}^{2}$ intersect.

In order to prove Theorem 1, we extend a 2-dimensional conic bundle over $\mathbb{P}_{\mathbb{Q}}^{1}$ to a 3 -dimensional conic bundle over $\mathbb{P}_{\mathbb{Z}}^{1}$ and argue as above. The answer seems to depend on subtle properties of $S \rightarrow \mathbb{P}_{\mathbb{Q}}^{1}$. In particular, the branch divisor $B_{S} \subset \mathbb{P}_{\mathbb{Q}}^{1}$ alone is not enough to decide what happens; see Example 21. 


\section{Surface conic bundles}

Fix an arbitrary field $k$. Assume for simplicity that char $k \neq 2$. Let $S$ be a smooth surface over $k$ and $S \rightarrow \mathbb{P}^{1}$ a minimal conic bundle. The number of singular geometric fibers, which is the degree of the branch locus $B_{S}$, is denoted by $\delta(S)$. A typical fiber is denoted by $F$. Thus $\left(K_{S}^{2}\right)=8-\delta(S)$ and $\left(K_{S} \cdot F\right)=-2$.

Lemma 9. Let $k$ be a field and $\pi: S \rightarrow \mathbb{P}^{1}$ a minimal conic bundle. Fix a natural number $m$ such that $\delta(S)>12+6 m$. Assume that $-K_{S}+m F$ is pseudo-effective.

Then there is a unique irreducible curve $C \subset S$ such that $\left(C^{2}\right)<0$. Furthermore, $\pi: C \rightarrow \mathbb{P}^{1}$ has degree 2 and $\left|-K_{S}+m F\right|=C+|b F|$ for some $b \geq 0$. In particular, $-K_{S}+m F$ is effective.

Proof. By assumption there is a sequence of effective $\mathbb{Q}$-divisors $D_{t}$ converging to $-K_{S}+m F$. Note that $\left(-K_{S}+m F\right)^{2}=8-\delta(S)+4 m<0$, hence $\left(D_{t}^{2}\right)<0$ for some $t$. So there is a $k$-irreducible component $C \subset \operatorname{Supp} D_{t}$ with $C^{2}<0$. Thus the cone of curves is generated by $C$ and a fiber $F$.

Write $C \sim_{\mathbb{Q}} a\left(-K_{S}+m^{\prime} F\right)$. Here $a$ is an integer but $m^{\prime}$ could be rational. The degree of the dualizing sheaf of $C$ is

$$
\operatorname{deg} \omega_{C}=2 p_{a}(C)-2=C\left(C+K_{S}\right)=a(a-1)\left(K_{S}^{2}\right)+2 a(2 a-1) m^{\prime} .
$$

Since $C_{\bar{k}}$ has at most $2 a$ irreducible components, $\operatorname{deg} \omega_{C} \geq-4 a$, hence

$$
a(a-1)\left(K_{S}^{2}\right)+2 a(2 a-1) m^{\prime} \geq-4 a .
$$

If $a \geq 2$ then this rearranges to

$$
\delta(S)-8=-\left(K_{S}^{2}\right) \leq\left(4+\frac{2}{a-1}\right) m^{\prime}+\frac{4}{a-1} \leq 6 m^{\prime}+4 .
$$

Note that $-K_{S}+m F \sim_{\mathbb{Q}} \frac{1}{a} C+\left(m-m^{\prime}\right) F$, hence $m^{\prime} \leq m$ since $-K_{S}+m F$ is pseudo-effective. Thus, if $\delta(S)>12+6 m$ then $a=1$.

Note that we also could have used Bend-and-break (as stated in [Kol96, II.5.5.3]) to show that $a \leq 2$.

Example 10. There are minimal conic bundles $S \rightarrow \mathbb{P}^{1}$ with $\delta(S)=12$ for which $-2 K_{S}$ is effective but $-K_{S}$ is not effective.

To construct such examples, let $Q \subset \mathbb{P}^{2}$ be an irreducible degree 4 point. Then $B_{Q} \mathbb{P}^{2} \rightarrow \mathbb{P}^{1}$ is a minimal conic bundle with with $\delta=3$. The exceptional curves give a conjugate set of 4 sections, each with self-intersection -1 .

Pull it back by a general degree $r$ map $\mathbb{P}^{1} \rightarrow \mathbb{P}^{1}$ to get $S \rightarrow \mathbb{P}^{1}$. We get a minimal conic bundle $S \rightarrow \mathbb{P}^{1}$ with $\delta(S)=3 r$ and a conjugate set of 4 sections, each with self-intersection $-r$. The sum of these 4 sections is in $\left|-2 K_{S}+(r-4) F\right|$. Thus the bound $\delta(S)>12+6 m$ in Lemma 9 is sharp.

(It is interesting to note that for $r=4$ contracting the 4 sections gives $S \rightarrow T$ where $T$ is a singular Enriques surface. It has 4 singular points and $2 K_{T} \sim 0$.)

Corollary 11. Let $k$ be a field and $S \rightarrow \mathbb{P}^{1}$ a minimal conic bundle. Assume that $\delta(S)>12+$ $6 m$. Then $-K_{S}+m F$ is birationally pseudo-effective iff $S$ contains a double section $C \subset S$ whose normalization has genus $\leq m+1$.

Proof. Assume that $S \rightarrow S^{\prime}$ is birational and $-K_{S^{\prime}}+m F^{\prime}$ is pseudo-effective. By [Isk67] (see also Theorem 27), $S^{\prime}$ is another minimal conic bundle with the same $\delta, S \rightarrow \mathbb{P}^{1}$ and $S^{\prime} \rightarrow \mathbb{P}^{1}$ have the same generic fiber and $-K_{S^{\prime}}+m^{\prime} F^{\prime}$ is linearly equivalent to an irreducible double section $C^{\prime}$ for some $m^{\prime} \leq m$ by Lemma 9. Thus $C$ is obtained as the birational transform of $C^{\prime}$. Its normalization has genus $\leq m+1$ by Lemma 12 . 
Conversely, let $C \subset S$ be a double section. We can resolve the singularities of $C$ by performing elementary transformations at its singular points; see Paragraph 26. (Clearly, these points are on smooth fibers.) At the end we have $C^{\prime} \subset S^{\prime}$ and $C^{\prime}$ is smooth. If $p_{a}\left(C^{\prime}\right) \leq m+1$ then $C^{\prime}$ is a sub-curve of $\left|-K_{S^{\prime}}+m F^{\prime}\right|$ by Lemma 12 .

Lemma 12. Let $C \subset S$ be a double section. Then $C$ is a sub-curve of $\left|-K_{S}+m F\right|$ iff $p_{a}(C) \leq m+1$.

Proof. $C \sim-K_{S}+b F$ for some $b \in \mathbb{Z}$. Thus $2 p_{a}(C)-2=C\left(C+K_{S}\right)=b(C \cdot F)=2 b$ hence $b \leq m$ iff $p_{a}(C) \leq m+1$.

\section{Threefold conic bundles}

We use Corollary 11 to prove a similar result for 3-dimensional conic bundles.

Theorem 13. Let $\pi: X \rightarrow \mathbb{P}^{2}$ be a smooth, minimal conic bundle over a field of characteristic $\neq 2,3,5$ whose branch curve $B_{X} \subset \mathbb{P}^{2}$ has degree $\geq 19$. The following are equivalent.

1. $-K_{X}$ is birationally pseudo-effective.

2. There is a generically finite double section $D \subset X$ with normalization $\tau: \bar{D} \rightarrow D$ such that the branch curve $B_{D}$ of $\pi \circ \tau: \bar{D} \rightarrow \mathbb{P}^{2}$ has degree $\leq 6$.

3. $X$ is birational to a smooth, minimal conic bundle $\pi^{\prime}: X^{\prime} \rightarrow \mathbb{P}^{2}$ (with the same branch curve) such that $\left|-K_{X^{\prime}}\right| \neq \emptyset$.

Proof. Assume that $\phi: X \rightarrow X^{\prime}$ shows that $-K_{X}$ is birationally pseudo-effective. By Complement 28 , we may choose $X^{\prime}$ such that $\pi^{\prime}: X^{\prime} \rightarrow \mathbb{P}^{2}$ is also a conic bundle with the same branch curve. Write $-K_{X^{\prime}} \sim_{\mathbb{Q}} \Delta^{\prime}$ where $\Delta^{\prime}$ is pseudo-effective.

Let $L \subset \mathbb{P}^{2}$ be the generic line. By restriction we get $\pi_{S}^{\prime}: S^{\prime} \rightarrow L$. Since $-K_{S^{\prime}}+F=-\left.K_{X^{\prime}}\right|_{S^{\prime}}$, we see that $-K_{S^{\prime}}+\left.F \sim_{\mathbb{Q}} \Delta^{\prime}\right|_{S^{\prime}}$ is pseudo-effective. Moreover, if $\Delta_{t}^{\prime}$ is a sequence of $\mathbb{Q}$-effective divisors converging to $\Delta^{\prime}$ then $\left.\Delta_{t}^{\prime}\right|_{S^{\prime}}$ is a sequence of $\mathbb{Q}$-effective divisors converging to $\left.\Delta^{\prime}\right|_{S^{\prime}}$.

Thus, by Lemma $9,\left.\Delta_{t}^{\prime}\right|_{S^{\prime}}$ has an irreducible component $C^{\prime} \subset S^{\prime}$ with negative self-intersection and its normalization has genus $\leq 2$ by Lemma 12 . Since $L$ is the generic line, $C^{\prime}$ is the restriction of an irreducible component $D^{\prime}$ of $\Delta_{t}^{\prime}$. Thus $D^{\prime}$ is a double section and its birational transform on $X$ is a double section $D \subset X$. Furthermore, we know that the preimage of $L$ in the normalization $\tau: \bar{D} \rightarrow D$ has genus $\leq 2$. Thus $L$ intersects the branch curve of $\pi \circ \tau: \bar{D} \rightarrow \mathbb{P}^{2}$ in $\leq 6$ points. This shows that $(1) \Rightarrow(2)$.

Assume next that $\pi$ has a double section $D \subset X$ as in (2). By Lemma 14, there is a sequence of elementary transformations $X \rightarrow X^{\prime}$ such that the branch curve of $\pi^{\prime}: D^{\prime} \rightarrow \mathbb{P}^{2}$ has degree $\leq 6$. By Lemma 15 this implies that $D^{\prime}$ is a sub-divisor of $\left|-K_{X^{\prime}}\right|$. Thus $(2) \Rightarrow(3)$ and $(3) \Rightarrow(1)$ holds by definition.

Lemma 14. Let $\pi: X \rightarrow Z$ be a conic bundle. Assume that $X$ and $Z$ are smooth and $\operatorname{dim} Z=2$. Let $D \subset X$ be a generically finite double section. Then there is a sequence of elementary transformations $X \rightarrow X^{\prime}$ such that the resulting $D^{\prime} \subset X^{\prime}$ is normal, except possibly along finitely many fibers.

Proof. Let $C \subset \operatorname{Sing} D$ be a curve not contained in a fiber of $\pi$. Let $F^{\prime} \subset F \subset X$ be an irreducible component of a fiber that is not contained in $D$ but meets $C$ at a point $p$. Since $D$ is singular at $p$, the local intersection number $\left(D \cdot F^{\prime}\right)_{p}$ is at least 2 . Since $D$ is a double section, $(D \cdot F) \leq 2$. Thus $F$ is irreducible and $D \cap F=\{p\}$, hence $\pi_{C}: C \rightarrow \pi(C)$ is birational. As we discuss in Paragraph 26 , the elementary transformation centered at $C$ decreases the degree of the branch curve of $D \rightarrow \mathbb{P}^{2}$. After finitely many such steps we get $\pi^{\prime}: X^{\prime} \rightarrow Z$ such that $D^{\prime} \subset X^{\prime}$ is normal, except possibly along finitely many fibers. 
Lemma 15. Let $\pi: X \rightarrow \mathbb{P}^{2}$ be a minimal conic bundle and $D \subset X$ a generically finite double section. Then $D$ is a sub-divisor of $\left|-K_{X}\right|$ iff the branch curve $B_{D} \subset \mathbb{P}^{2}$ of $\pi_{D}: D \rightarrow \mathbb{P}^{2}$ has degree $\leq 6$.

Proof. Since $X$ is minimal, we know that $D \sim-K_{X}+b \pi^{*} H$ for some $b \in \mathbb{Z}$ where $H$ is the class of a line in $\mathbb{P}^{2}$. Thus $K_{D} \sim b \pi_{D}^{*} H$.

Let $D \rightarrow D^{\prime} \rightarrow \mathbb{P}^{2}$ denote the Stein factorization. Then $K_{D} \sim b \pi_{D}^{*} H$ pushes forward to $K_{D^{\prime}} \sim$ $b \pi_{D^{\prime}}^{*} H$. By the Hurwitz formula $K_{D^{\prime}} \sim \pi_{D^{\prime}}^{*}\left(K_{\mathbb{P}^{2}}+\frac{1}{2} B_{D}\right)$. Thus $b \leq 0$ iff $\operatorname{deg} B_{D} \leq 6$.

\section{K3 surfaces on conic bundles}

In order to prove Theorem 3, it remains to show that (13.2) does not hold for suitable branch curves $B \subset \mathbb{P}^{2}$. Example 20 shows that there are conic bundles with high degree smooth branch curve for which $\left|-K_{X}\right|$ does contain a K3 surface. Thus we need to focus on more subtle properties of $B$. We present 2 approaches. The first uses branch curves with many nodes; we prove that all the nodes have to lie on a sextic curve. This leads to quite explicit examples starting with $\operatorname{deg} B=12$; see Corollary 17.

Note also that, in a flat family of conic bundles, double sections are parametrized by countably many components of the Chow variety. Thus if we find one conic bundle without certain type of double sections then the very general conic bundle also has no double sections of the same type.

The other approach proves that there has to be a sextic curve that is everywhere tangent to $B_{X}$. This is easily seen to be a non-empty Zariski open condition for $\operatorname{deg} B \geq 15$, though I could not write down explicit examples. As pointed out by [Sar82, Sec.5], the results of [AM72, Sec.3] imply that every smooth plane curve of degree $\geq 3$ is the branch curve of a minimal conic bundle.

Lemma 16. Let $\pi: X \rightarrow Z$ be a minimal, smooth conic bundle with branch curve $B_{X}$. Let $D \subset X$ be a double section with normalization $\tau: \bar{D} \rightarrow D$ and $B_{D} \subset Z$ the branch curve of $\left.\pi\right|_{D} \circ \tau: \bar{D} \rightarrow Z$. Then $\operatorname{Sing}\left(B_{X}\right) \subset B_{D}$.

Proof. If $p \notin B_{D}$ then both of the étale local branches of $\bar{D} \rightarrow Z$ give étale local sections of $\pi$. As we discuss in Claim 25.5, there are no étale local sections over the nodes of $B_{X}$ for $X$ smooth.

Corollary 17. Let $X \subset \mathbb{P}_{\mathbf{x}}^{2} \times \mathbb{P}_{\mathbf{y}}^{2}$ be given by an equation of bidegree $(d, 2)$

$$
\sum_{i} g_{i}\left(x_{0}, x_{1}, x_{2}\right) y_{i}^{2}=0 .
$$

Assume that the curves $B_{i}:=\left(g_{i}\left(x_{0}, x_{1}, x_{2}\right)=0\right) \subset \mathbb{P}_{\mathbf{x}}^{2}$ are smooth and they intersect each other transversally in $3 d^{2}$ distinct points.

Let $D \subset X$ be a double section and $B_{D} \subset \mathbb{P}_{\mathbf{x}}^{2}$ the branch curve of $\left.\pi\right|_{D} \circ \tau: \bar{D} \rightarrow Z$. Then $\operatorname{deg} B_{D} \geq 2 d$.

Proof. The assumptions imply that $X$ is smooth. The branch curve of $X$ is $B_{X}=B_{0}+B_{1}+B_{2}$. By Lemma $16, B_{D}$ passes through all the nodes of $B$. In particular, $B_{D}$ intersects $B_{0}$ in the $2 d^{2}$ points $B_{0} \cap\left(B_{1} \cup B_{2}\right)$. Thus deg $B_{D} \geq 2 d$ unless $B_{0} \subset B_{D}$. We can repeat the argument for $B_{1}$ to get that $\operatorname{deg} B_{D} \geq 2 d$ unless $B_{0}+B_{1} \subset B_{D}$. The letter case also implies that $\operatorname{deg} B_{D} \geq 2 d$.

For conic bundles with smooth branch curves we have a less precise condition. (The 2 results are closely related since passing through a node guarantees that the intersection multiplicity is $\geq 2$.)

Proposition 18. Let $\pi: X \rightarrow \mathbb{P}^{2}$ be a smooth, minimal conic bundle with branch locus $B_{X} \subset \mathbb{P}^{2}$. Assume that there is no reduced curve $C \subset \mathbb{P}^{2}$ of degree $\leq 6$ such that all points of $C \cap B_{X}$ have intersection multiplicity $\geq 2$. Then $-K_{X}$ is not effective. 
Proof. Assume to the contrary that $-K_{X} \sim D$ where $D$ is an effective $\mathbb{Z}$-divisor. Since $X$ is minimal, there are no rational sections. Thus there is a unique irreducible component $D_{0} \subset D$ that is a double section of $\pi$. Let $\tau: \bar{D}_{0} \rightarrow D_{0}$ denote the normalization and let $B_{D} \subset \mathbb{P}^{2}$ be the branch curve of $\left.\tau \circ \pi\right|_{D_{0}}: \bar{D}_{0} \rightarrow \mathbb{P}^{2}$. Note that $D_{0} \sim-K_{X}-m H$ for some $m \geq 0$ and $\operatorname{deg} B_{D} \leq 6$ by Lemma 15 .

We claim that all points of $B_{D} \cap B_{X}$ have intersection multiplicity $\geq 2$. To see this, assume to the contrary that there is a point $p \in B_{D} \cap B_{X}$ where $B_{D}, B_{X}$ are smooth and intersect transversally. In a neighborhood of $p$ the surface $D_{0}$ is a smooth double cover ramified along $B_{D}$. Then $\left(\left.\pi\right|_{D_{0}}\right)^{-1}\left(B_{X}\right)$ is a smooth double cover of $B_{X}$ ramified at $p$. On the other hand, $\left(\left.\pi\right|_{D_{0}}\right)^{-1}\left(B_{X}\right)=D_{0} \cap \pi^{-1}\left(B_{X}\right)$ hence its normalization factors through the étale cover $\tilde{B}_{X} \rightarrow B_{X}$. This is a contradiction. (This argument in fact shows that these intersection multiplicities are even.)

Next we check that the assumptions of Proposition 18 hold for a general branch curve of degree $\geq 15$.

Lemma 19. Let $B \subset \mathbb{P}^{2}$ be a general curve of degree $d$. Then there is no reduced curve $C \subset \mathbb{P}^{2}$ of degree $<\frac{1}{2} d-1$ such that all points of $C \cap B$ have intersection multiplicity $\geq 2$.

Proof. Fix a reduced curve $C$ and let $L$ be a line bundle of degree $m$. Let $W(L) \subset H^{0}(C, L)$ denote the subvariety consisting of sections without simple zeros. Note that any $s \in W(L)$ has at most $\frac{1}{2} m$ zeros. The map that sends a section of $L$ to its set of zeros has 1-dimensional fibers. Thus $\operatorname{dim} W(L) \leq \frac{1}{2} m+1$.

Fix a reduced plane curve $C \subset \mathbb{P}^{2}$ of degree $c$. Let $W(C, d)$ denote the set of degree $d$ curves $B$ such that all points of $C \cap B$ have intersection multiplicity $\geq 2$. Applying the above estimate to $L=\left.\mathcal{O}_{\mathbb{P}^{2}}(d)\right|_{C}$ we get that $\operatorname{dim} W(C, d) \leq \frac{1}{2} c d+\left(\begin{array}{c}d-c+2 \\ 2\end{array}\right)$. Next let $W(d)$ denote the set of degree $d$ curves $B$ such that all points of $C \cap B$ have intersection multiplicity $\geq 2$ for some curve $C$ of degree c. Then

$$
\operatorname{dim} W(d) \leq \frac{1}{2} c d+\left(\begin{array}{c}
d-c+2 \\
2
\end{array}\right)+\left(\begin{array}{c}
c+2 \\
2
\end{array}\right)-1 .
$$

After expanding the binomials we see that

$$
\operatorname{dim} W(d)<\left(\begin{array}{c}
d+2 \\
2
\end{array}\right)-1 \quad \text { for } d>2 c+2 .
$$

(Note that the bound $\frac{1}{2} d-1$ is sharp for $d=4$ but one should be able to prove slightly better bounds for larger values of $d$.)

Example 20. Let $Z$ be a smooth variety and $E$ a rank 3 vector bundle one $Z$. Set $P:=\mathbb{P}_{Z}(E)$ with projection $\pi: P \rightarrow Z$ and note that $\omega_{P / Z} \cong \mathcal{O}_{P}(-3) \otimes \pi^{*} \operatorname{det} E$. Let $L$ be a line bundle on $Z$ and $X \subset P$ the zero set of a section of $\mathcal{O}_{P}(2) \otimes \pi^{*} L$. Then $\left.\omega_{X} \cong\left(\mathcal{O}_{P}(-1) \otimes \pi^{*}(L \otimes \operatorname{det} E)\right)\right|_{X}$ and $\pi_{*}\left(\omega_{X}^{-1}\right) \cong\left(\omega_{Z} \otimes L \otimes \operatorname{det} E\right)^{-1} \otimes E$.

Note that $H^{0}\left(P, \mathcal{O}_{P}(2) \otimes \pi^{*} L\right)=H^{0}\left(Z, L \otimes \operatorname{Sym}^{2} E\right)$ and $H^{0}\left(X, \mathcal{O}_{X}\left(-K_{X}\right)\right)=H^{0}\left(Z,\left(L\left(K_{Z}\right) \otimes\right.\right.$ $\left.\operatorname{det} E)^{-1} \otimes E\right)$. We would like $X$ to be smooth, this suggests that $L \otimes \operatorname{Sym}^{2} E$ should be generated by global sections, hence $L$ should be positive. By contrast, the condition $\left|-K_{X}\right| \neq \emptyset$ suggests that $L$ should be negative. It seems that both of these can be satisfied only if $E$ is rather unstable.

For example, take $Z=\mathbb{P}^{2}$, set $E=\mathcal{O}_{\mathbb{P}^{2}}(c) \oplus \mathcal{O}_{\mathbb{P}^{2}}(3) \oplus \mathcal{O}_{\mathbb{P}^{2}}$ where $c>3$ and $L=\mathcal{O}_{\mathbb{P}^{2}}$. Then $L \otimes \operatorname{Sym}^{2} E$ is generated by global sections, so a general section gives a smooth conic bundle $X$. Furthermore,

$$
\pi_{*}\left(\omega_{X}^{-1}\right) \cong \mathcal{O}_{\mathbb{P}^{2}}(3-3-c) \otimes E \cong \mathcal{O}_{\mathbb{P}^{2}} \oplus \mathcal{O}_{\mathbb{P}^{2}}(3-c) \oplus \mathcal{O}_{\mathbb{P}^{2}}(-c)
$$


has a unique section. Thus $X \rightarrow \mathbb{P}^{2}$ is a conic bundle such that $\left|-K_{X}\right|$ contains a unique K3 surface. It is obtained by intersecting $X$ with the divisor in $P$ corresponding to the $\mathcal{O}_{\mathbb{P}^{2}}(3) \oplus \mathcal{O}_{\mathbb{P}^{2}}$ summand of $E$. The branch curve of $X$ is given by the equation

$$
\operatorname{det}\left(\begin{array}{ccc}
g_{2 c} & g_{c+3} & g_{c} \\
g_{c+3} & g_{6} & g_{3} \\
g_{c} & g_{3} & g_{0}
\end{array}\right)=0
$$

where $g_{i}:=g_{i}(x, y, z)$ denotes a homogeneous polynomial of degree $i$. (We can thus assume that $g_{0}=1$.) Eliminating $g_{c}, g_{3}$ in the last row writes $g_{0}$ times the determinant, hence the equation of $B_{X}$, in the form

$$
\left(g_{6} g_{0}-g_{3}^{2}\right)\left(g_{2 c} g_{0}-g_{c}^{2}\right)-\left(g_{c+3} g_{0}-g_{c} g_{3}\right)^{2}=0 .
$$

Thus the degree 6 curve $g_{6} g_{0}-g_{3}^{2}=0$ intersects $B_{X}$ at the points where $g_{c+3} g_{0}-g_{c} g_{3}=0$ and all intersection multiplicities are even; as needed in Lemma 18.

\section{Examples of conic bundle surfaces}

Working backwards from Theorem 3 we see that there are surface conic bundles $S \rightarrow \mathbb{P}^{1}$ over $\mathbb{C}(s, t)$ such that $-K_{S}$ is not birationally $\mathbb{Q}$-effective. We will exhibit similar examples over $\mathbb{Q}$ and $\mathbb{Q}_{p}$.

Example 21. Let $k$ be a field and $S \rightarrow \mathbb{P}^{1}$ a conic bundle over $k$ that becomes trivial after a quadratic extension $k^{\prime} \supset k$. Then $S_{k^{\prime}}$ has a section $C^{\prime}$, thus $S$ has a conjugate pair of sections $C$. The normalization of $C$ has $p_{a}=-1$. Thus Corollary 11 implies that $S \rightarrow \mathbb{P}^{1}$ is birational to a conic bundle $S^{\prime} \rightarrow \mathbb{P}^{1}$ such that $-K_{S^{\prime}}$ is effective. (Typically the base locus of $\left|-K_{S}\right|$ consists of the disjoint conjugate sections and the moving part of 2 fibers.)

There are such examples over $\mathbb{Q}$, even with arbitrary branch locus. Let $g(s) \in \mathbb{Q}[s]$ be a polynomial of degree $2 d$ with simple roots only. Choose a prime $p$ such that $\sqrt{p}$ is not contained in the splitting field of $g$. Let $S_{g} \rightarrow \mathbb{P}^{1}$ be the projective model of the surface

$$
\left(g(s) z^{2}=x^{2}-p y^{2}\right) \subset \mathbb{P}_{x y z}^{1} \times \mathbb{A}_{s}^{1} .
$$

Then $S_{g} \rightarrow \mathbb{P}^{1}$ is minimal and the singular fibers lie exactly over the roots of $g(s)$. Thus there are surface conic bundles $S \rightarrow \mathbb{P}^{1}$ with $\delta(S)$ arbitrarily large and branch locus $B_{S}$ in general position for which $-K_{S}$ is birationally effective.

Note also that the above argument implies that $-K_{S}$ is birationally effective for every conic bundle over $\mathbb{R}$ since every surface conic bundle over $\mathbb{C}$ has a section. The situation over finite fields is unclear to me.

The examples where $-K_{S}$ is not birationally $\mathbb{Q}$-effective rest on the following observation.

Lemma 22. Let $Z$ be a 2-dimensional regular scheme and $\pi: X \rightarrow Z$ a conic bundle. Let $C \subset Z$ be an irreducible 1-dimensional subscheme such that $X_{C}:=\pi^{-1}(C) \rightarrow C$ has no rational sections. Let $W \subset C$ be the set of points $z \in C$ such that $X_{z}$ is a double line and $X$ is regular along $X_{z}$. Let $D \subset X$ be a double section with normalization $\tau: \bar{D} \rightarrow D$ and $B_{D} \subset Z$ the branch locus of $\left.\pi\right|_{D} \circ \tau: \bar{D} \rightarrow Z$.

Then $C \not \subset B_{D}$ but $W \subset B_{D}$.

Proof. If $C \subset B_{D}$ then $\operatorname{red}\left(D \cap X_{C}\right)$ is a rational section of $X_{C} \rightarrow C$. This is contrary to our assumptions and $W \subset B_{D}$ follows from Claim 25.3. 
Example 23. We will apply Lemma 22 with $Z=\mathbb{P}_{\mathbb{Z}_{p}}^{1}$ and $C=\mathbb{P}_{\mathbb{F}_{p}}^{1}$ for some prime $p \geq 3$. Fix a natural number $d$ and set $P:=\mathbb{P}_{Z}\left(\mathcal{O}_{Z} \oplus \mathcal{O}_{Z}(d) \oplus \mathcal{O}_{Z}(d+1)\right)$. The conic bundle $X \subset P$ will be given by a section of $\mathcal{O}_{P}(2)$. For simplicity we choose a section of $\mathcal{O}_{Z}+\mathcal{O}_{Z}(2 d) \oplus \mathcal{O}_{Z}(2 d+2) \subset \pi_{*} \mathcal{O}_{P}(2)$. Choosing an affine coordinate $s$ on $\mathbb{P}^{1}$, one can give such an $X$ by an equation

$$
X:=\left(z^{2}=a(s) x^{2}+b(s) y^{2}\right) \quad \text { where } \quad \operatorname{deg} a(s)=2 d, \operatorname{deg} b(s)=2 d+2 .
$$

We choose $a(s)$ and $b(s)$ as follows.

1. $\bar{a}(s)$ has only simple zeros where ${ }^{-}$denotes reduction $\bmod p$,

2. $b(s)=\left(s^{2}-1\right) a(s)+p c(s)$ for some $c(s)$,

3. $s^{2}-1, \bar{a}(s), \bar{c}(s)$ are pairwise relatively prime and

4. $\bar{a}(1)$ is not a square in $\mathbb{F}_{p}$.

Claim 23.5. Let $\pi: X_{\mathbb{Q}_{p}} \rightarrow \mathbb{P}_{\mathbb{Q}_{p}}^{1}$ be the generic fiber of the above $X \rightarrow Z$ and $D_{\mathbb{Q}_{p}} \subset X_{\mathbb{Q}_{p}}$ a double section with normalization $\tau: \bar{D}_{\mathbb{Q}_{p}} \rightarrow D_{\mathbb{Q}_{p}}$. Then $g\left(\bar{D}_{\mathbb{Q}_{p}}\right) \geq d-1$.

Proof. The closure of $D_{\mathbb{Q}_{p}}$ gives a double section $D \subset X$. Let $B_{D} \subset Z$ be the branch locus of $\left.\pi\right|_{D} \circ \tau: \bar{D} \rightarrow Z$. We aim to apply Lemma 22 .

Let $\alpha$ be a root of $a(s)$. Near $\alpha$ the equation of $X$ has the form

$$
z^{2}=(s-\alpha) u_{1} x^{2}+\left((s-\alpha) u_{2}+p u_{3}\right) y^{2},
$$

where the $u_{i}$ are units. Thus the fiber $X_{\alpha}$ is a double line and $X$ is regular along $X_{\alpha}$ by (25.5). Over the point $(s=1) \in \mathbb{P}_{\mathbb{F}_{p}}^{1}$ the fiber is $\left(z^{2}=\bar{a}(1) x^{2}\right) \subset \mathbb{P}_{\mathbb{F}_{p}}^{2}$. Since $\bar{a}(1)$ is not a square, its only $\mathbb{F}_{p}$-point is $(0: 1: 0)$ where $\bar{X}$ is smooth. Hence $\bar{X} \rightarrow \mathbb{P}_{\mathbb{F}_{p}}^{1}$ has no sections. Thus Lemma 22 implies that

$$
2 g\left(\bar{D}_{\mathbb{Q}_{p}}\right)+2=\operatorname{deg} B_{D} \geq \operatorname{deg} a(s)=2 d .
$$

The choices (23.1-23.4) can be satisfied for $a(s), b(s) \in \mathbb{Q}[s]$, hence we proved the following more precise form of Theorem 1.

Corollary 24. For every $g$ there are conic bundles $S \rightarrow \mathbb{P}^{1}$ defined over $\mathbb{Q}$ with $\delta(S)=4 g+6$ and such that every double section of $S$ has geometric genus $\geq g$.

For $g=2$ this gives conic bundles $S \rightarrow \mathbb{P}^{1}$ with $\delta(S)=14$ defined over $\mathbb{Q}$ such that $-K_{S}$ is not birationally pseudo-effective.

\section{Birational maps of conic bundles}

We summarize the results on birational maps of conic bundles that we used. As before, all residue characteristics are assumed to be $\neq 2$.

25. (Extending conic bundles) We will need to understand the following

Problem 25.1. Let $Z$ be a regular surface, $W \subset Z$ a finite subset and $\pi^{0}: X^{0} \rightarrow Z^{0}:=Z \backslash W$ a conic bundle with branch locus $B_{X}^{0}$. We would like to extend $\pi^{0}: X^{0} \rightarrow Z^{0}$ to a conic bundle $\pi: X \rightarrow Z$ and control the singularities of $X$ in terms of $B_{X}$. (This is also interesting if $\operatorname{dim} Z>2$ but the 2 -dimensional case is simpler.) 
We may assume that $W=\{p\}$ is a single point and $Z$ is local. The push-forward $E^{0}:=\pi_{*}^{0} \omega_{x^{0} / Z^{0}}^{-1}$ is a locally free sheaf of rank 3 . Set $E:=j_{*} E^{0}$ where $j: Z^{0} \hookrightarrow Z$ is the natural injection. Then $E$ is a reflexive sheaf but, since $Z$ is regular and 2-dimensional, $E$ is locally free, hence free. Set $P:=\mathbb{P}_{Z}(E)$ and let $X \subset P$ be the closure of $X^{0} \subset P^{0}$. Choose an isomorphism $P \cong \mathbb{P}_{Z}^{2}$, then $X$ is given by an equation

$$
\sum_{i j} g_{i j}\left(z_{1}, z_{2}\right) x_{i} x_{j}=0
$$

where $g_{i j}\left(z_{1}, z_{2}\right) \in \mathcal{O}_{Z}$. The scheme structure of the branch locus is defined by

$$
\operatorname{det}\left(g_{i j}\left(z_{1}, z_{2}\right)\right)=0 \text {. }
$$

The worst case is when the central fiber $X_{p}$ equals $\mathbb{P}^{2}$; thus $\pi: X \rightarrow Z$ is not even equidimensional. If this happens then all the $g_{i j}$ vanish at $p$ so $\operatorname{det}\left(g_{i j}\left(z_{1}, z_{2}\right)\right) \in m_{p}^{3}$. Thus $B_{X}$ has a triple (or higher) point at $p$. Otherwise the central fiber $X_{p}$ is conic.

$X_{p}$ is smooth iff $p \notin B_{X}$.

Next assume that $X_{p}$ is a pair if lines. Possibly after a quadratic residue field extension, in suitable formal coordinates we can diagonalize the equation of $X$ as

$$
x_{0}^{2}=x_{1}^{2}+f\left(z_{1}, z_{2}\right) x_{2}^{2} \quad \text { and } \quad B_{X}=\left(f\left(z_{1}, z_{2}\right)=0\right)
$$

where $f(0,0)=0$. We see that $X$ is regular along $X_{p}$ iff $f \notin\left(z_{1}, z_{2}\right)^{2}$; that is, iff $B_{X}$ is regular at $p$. Furthermore, $\left(z_{1}, z_{2}\right) \mapsto\left(1: 1: 0 ; z_{1}, z_{2}\right)$ is a formal section.

Finally consider the case when $X_{p}$ is a double line. Then $\operatorname{det}\left(g_{i j}\left(z_{1}, z_{2}\right)\right) \in m_{p}^{2}$ and $B_{X}$ has a double (or higher) point at $p$. If the branch curve has a node then, after a quadratic residue field extension, in suitable formal coordinates we can write $X$ as

$$
X=\left(x_{0}^{2}=z_{1} x_{1}^{2}+z_{2} x_{2}^{2}\right) .
$$

The fiber of the projection $\pi: X \rightarrow \mathbb{A}_{\mathbf{z}}^{2}$ is a double line over the origin, a pair of lines over the coordinate axes $\left(z_{1} z_{2}=0\right)$ and smooth otherwise. In contrast with the previous nodal case, the equation has no solutions in the quotient field of the completion of $\mathcal{O}_{z, Z}$; see Claim 25.5.

We have thus proved the following.

Claim 25.4. Let $Z$ be a regular surface whose residue characteristics are $\neq 2, B \subset Z$ a curve with only nodal singularities and $W \in Z$ a finite subset containing the nodes of $B$. Let $\pi^{0}: X^{0} \rightarrow Z \backslash W$ be a conic bundle with (scheme theoretic) branch locus $B \backslash W$. Then $X^{0}$ extends to a unique conic bundle $\pi: X \rightarrow Z$.

Furthermore, $X$ is regular iff the following holds: for every $p \in W$ that is a node of $B$, the projection $\pi: X \rightarrow Z$ has no sections over the quotient field of the strict henselization $\mathcal{O}_{p, Z}^{\mathrm{sh}}$ of the local ring $\mathcal{O}_{p, Z}$.

We also used the following well known result.

Claim 25.5. Let $(R, m=(s, t))$ be a regular local ring of dimension 2. Then $x_{0}^{2}=s x_{1}^{2}+t x_{2}^{2}$ has no nonzero solutions in the quotient field of $R$.

Proof. After clearing denominators, we may assume that the $x_{i}$ are in $R$. Let $c$ be the largest such that the $x_{i}$ are in $m^{c}$. We can thus write $x_{i}=p_{i}(s, t)+r_{i}$ where $p_{i}$ is a homogeneous polynomial of degree $c$ and $r_{i} \in m^{c+1}$. Then $x_{0}^{2} \in m^{2 c}$ but $s x_{1}^{2}+t x_{2}^{2} \in m^{2 c+1}$. Thus in fact $p_{0} \equiv 0$ and so $s p_{1}^{2}+t p_{2}^{2} \in m^{2 c+2}$. Thus $s p_{1}^{2}+t p_{2}^{2} \equiv 0$ hence $p_{1}$ and $p_{2}$ are both identically 0 . This is a contradiction.

26. (Elementary transformations of conic bundles) Let $\pi: X \rightarrow Z$ be a conic bundle over a regular surface and $C \subset Z$ a regular curve such that $\pi$ is smooth over $C$ and $s: C \rightarrow X_{C}$ a section of $X_{C} \rightarrow C$. We first blow up $s(C) \subset X$ and then contract the birational transform of $X_{C}$. We 
get another conic bundle $\pi^{(s)}: X^{(s)} \rightarrow Z$. The rational map $X \rightarrow X^{(s)}$ is called the elementary transformation with center $s(C)$.

Next consider the case when $C \subset Z$ is a geometrically reduced curve, $\pi$ is smooth over the generic points of $C$ and $s: C \rightarrow X_{C}$ is a rational section of $X_{C} \rightarrow C$. Then there is a finite subset $W \subset Z$ such that $Z^{0}:=Z \backslash W, C^{0}:=C \backslash W$ and $X^{0}:=X \backslash \pi^{-1}(W)$ satisfy the previous assumptions. We can thus construct the elementary transformation $\pi^{0,(s)}: X^{0,(s)} \rightarrow Z^{0}$ of $\pi^{0}: X^{0} \rightarrow Z^{0}$ with center $s\left(C^{0}\right)$. Finally, whenever possible, we use the method of Paragraph 25 to extend $\pi^{0,(s)}: X^{0,(s)} \rightarrow Z^{0}$ to a conic bundle $\pi^{(s)}: X^{(s)} \rightarrow Z$ called the elementary transformation with center $s(C)$.

Claim 26.1. Let $\pi: X \rightarrow Z$ be a conic bundle over a surface, $C \subset Z$ a geometrically reduced curve and $s: C \rightarrow X_{C}$ a rational section of $X_{C} \rightarrow C$. Assume that $X$ and $Z$ are regular and $\pi$ is smooth over the generic points of $C$.

Then the elementary transformation with center $s(C)$ exists and it is a conic bundle $\pi^{(s)}: X^{(s)} \rightarrow$ $Z$ such that $X^{(s)}$ is also regular and has the same branch curve as $X \rightarrow Z$.

The following result of [Sar80], whose idea goes back to [Isk67], describes birational transformations of conic bundles over the same base.

Claim 26.2. Let $\pi_{i}: X_{i} \rightarrow Z$ be smooth conic bundles over a smooth surface. Let $\phi: X_{1} \rightarrow X_{2}$ be a birational equivalence over $Z$. That is, the following diagram commutes

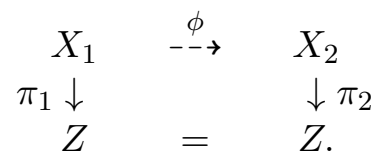

Then $\phi$ is a composite of elementary transformations.

The key result about birational maps of conic bundles is the following. The surface case is due to [Isk67]. (The minimal model program for surfaces over any field is established in [Mor82], thus the arguments of [Isk67] extend to any field.) The much harder 3-fold case is treated in [Sar80]. See [Cor95, Cor00] for more conceptual proofs.

Theorem 27. Let $\pi: X \rightarrow Z$ be a minimal conic bundle over a field of characteristic $\neq 2,3,5$ such that $X, Z$ are smooth and $B_{X}+4 K_{Z}$ is effective. Let $\pi^{\prime}: X^{\prime} \rightarrow Z^{\prime}$ be a Mori fiber space and $\phi: X^{\prime} \rightarrow X$ a birational map. Then $\pi^{\prime}$ is also a conic bundle, and there is a birational map $\phi_{Z}: Z^{\prime} \rightarrow Z$ such that the following diagram commutes

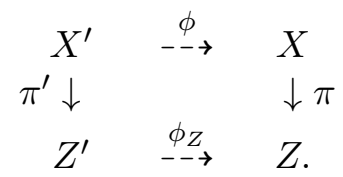

For our applications we need the following more precise version for which I could not find an explicit reference.

Complement 28. Assume in addition that $B_{X}+K_{Z}$ is ample. Then the diagram (2\%.1) can be factored as

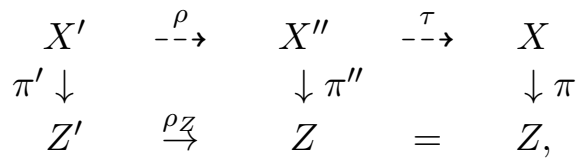

where $\tau: X^{\prime \prime} \rightarrow X$ is a composite of elementary transformations, $\rho: X^{\prime} \rightarrow X^{\prime \prime}$ is a birational contraction and $-K_{X^{\prime \prime}}=\rho_{*}\left(-K_{X^{\prime}}\right)$. 
Proof. Let $\pi: X \rightarrow Z$ be a conic bundle over a field of characteristic 0 such that $X, Z$ are smooth. Pick a point $z \in Z$ and let $p: Z_{1}:=B_{z} Z \rightarrow Z$ denote the blow-up. The pull-back $X \times{ }_{Z} Z_{1}$ is birational to a minimal conic bundle $\pi_{1}: X_{1} \rightarrow Z_{1}$. We want to describe its branch locus $B_{X_{1}}$. It is clear that $B_{X_{1}} \supset p_{*}^{-1} B_{X}$, the only question is what happens with the exceptional curve $E_{z}$. The following possibilities are listed in [Sar80, 2.4-2.5].

If $z \notin B_{x}$ then $X_{1}=X \times_{Z} Z_{1}$ hence $B_{X_{1}}=p_{*}^{-1} B_{X}$.

If $z \in B_{x}$ is a smooth point then $E_{z} \subset B_{X_{1}}$ iff the fiber $X_{z}$ irreducible. (Thus again $B_{X_{1}}=p_{*}^{-1} B_{X}$ if the base field is algebraically closed.)

If $z \in B_{x}$ is a singular point then $X_{1}=X \times_{Z} Z_{1}$ is singular. Using the local equation $x_{0}^{2}=$ $z_{1} x_{1}^{2}+z_{2} x_{2}^{2}$ we get $x_{0}^{2}=z_{1}^{\prime} x_{1}^{2}+z_{1}^{\prime} z_{2}^{\prime} x_{2}^{2}$. We can rewrite this as $z_{1}^{\prime}\left(x_{0}^{\prime}\right)^{2}=x_{1}^{2}+z_{2}^{\prime} x_{2}^{2}$ where $x_{0}^{\prime}=x_{0} / z_{1}^{\prime}$. Thus $B_{X^{\prime}}=p_{*}^{-1} B_{X}+E_{z}$. In all these cases we see that for any sequence of blow ups $p_{r}: Z_{r} \rightarrow Z$ we have

$$
\left.K_{Z_{r}}+B_{X_{r}}=p_{r}^{*}\left(K_{Z}+B_{X}\right)+\text { (effective exceptional divisor }\right)
$$

Applying this to a common resolution $Z^{\prime} \leftarrow Z_{r} \rightarrow Z$ we conclude that if $K_{Z}+B_{X}$ is ample then $\left(Z, B_{X}\right)$ is the unique log-canonical model of $\left(Z_{r}, B_{X_{r}}\right)$. In particular, the rational map $\phi_{Z}: Z^{\prime} \rightarrow Z$ in (27.1) is a morphism. This establishes the bottom row of (28.1).

In order to get the top row, let $A^{\prime}$ be an ample divisor on $Z^{\prime}$ and choose $m$ such that $\left|H^{\prime}\right|:=$ $\left|-K_{Z^{\prime}}+m\left(p^{\prime}\right)^{*} A^{\prime}\right|$ is a very ample linear system on $Z^{\prime}$. Its push-forward $\phi_{*}\left|H^{\prime}\right|$ is a mobile linear system consisting of rational double sections of $\pi$. Its base locus consists of some horizontal curves (that is curves $C_{i} \subset X$ such that $C_{i} \rightarrow \pi\left(C_{i}\right)$ is birational) and some vertical curves (that is curves that are contracted by $\pi$ ). By a small variation of Claim 26.2, a sequence of elementary transformations along the horizontal curves leads to a factorization

$$
\phi: X^{\prime} \stackrel{\rho}{-} \rightarrow X^{\prime \prime} \stackrel{\tau}{-\rightarrow} \rightarrow X
$$

where $\tau$ is a composite of elementary transformations and the base locus $\operatorname{Bs}\left(\rho_{*}\left|H^{\prime}\right|\right)$ is vertical. Thus $\rho^{-1}$ gives an injection $X^{\prime \prime} \backslash \operatorname{Bs}\left(\rho_{*}\left|H^{\prime}\right|\right) \hookrightarrow X^{\prime}$, hence it has no exceptional divisors. Thus $\rho$ is a birational contraction and therefore $-K_{X^{\prime \prime}}=\rho_{*}\left(-K_{X^{\prime}}\right)$.

\section{Acknowledgements}

I thank A. Corti, Y. Gongyo, A. Skorobogatov, C. Xu and the referees for helpful comments, corrections and references.

\section{References}

[AM72] M. Artin and D. Mumford, Some elementary examples of unirational varieties which are not rational, Proc. London Math. Soc. (3) 25 (1972), 75-95. MR-0321934

[Bea77] Arnaud Beauville, Variétés de Prym et jacobiennes intermédiaires, Ann. Sci. École Norm. Sup. (4) 10 (1977), no. 3, 309-391. MR-0472843

[CG13] Paolo Cascini and Yoshinori Gongyo, On the anti-canonical ring and varieties of Fano type, Saitama Math. J. 30 (2013), 27-38 (2013). MR-3203718

[Che04] Ivan Cheltsov, Conic bundles with a large discriminant, Izv. Math. 68 (2004), no. 2, 429-434. MR-2058006

[CK10] Ivan Cheltsov and Ilya Karzhemanov, Halphen pencils on quartic threefolds, Adv. Math. 223 (2010), no. 2, 594-618. MR-2565543 
[Cor95] Alessio Corti, Factoring birational maps of threefolds after Sarkisov, J. Algebraic Geom. 4 (1995), no. 2, 223-254. MR-1311348

[Cor00] _ Singularities of linear systems and 3-fold birational geometry, Explicit birational geometry of 3-folds, London Math. Soc. Lecture Note Ser., vol. 281, Cambridge Univ. Press, Cambridge, 2000, pp. 259-312. MR-1798984

[Isk67] V. A. Iskovskikh, Rational surfaces with a pencil of rational curves, Mat. Sb. (N.S.) 74 (116) (1967), 608-638. MR-0220734

[Isk87]__ On the rationality problem for conic bundles, Duke Math. J. 54 (1987), no. 2, 271294. MR-899398

[KM98] János Kollár and Shigefumi Mori, Birational geometry of algebraic varieties, Cambridge Tracts in Mathematics, vol. 134, Cambridge University Press, Cambridge, 1998, With the collaboration of C. H. Clemens and A. Corti, Translated from the 1998 Japanese original. MR-1658959

[KM17] János Kollár and Massimiliano Mella, Quadratic families of elliptic curves and unirationality of degree 1 conic bundles, Amer. J. Math. (2017), (to appear).

[Kol96] János Kollár, Rational curves on algebraic varieties, Ergebnisse der Mathematik und ihrer Grenzgebiete. 3. Folge., vol. 32, Springer-Verlag, Berlin, 1996. MR-1440180

[Kol13] _ Singularities of the minimal model program, Cambridge Tracts in Mathematics, vol. 200, Cambridge University Press, Cambridge, 2013, With the collaboration of Sándor Kovács. MR-3057950

[Kry14] I. Krylov, Rationally connected non-Fano type varieties, ArXiv e-prints (2014).

[KSC04] János Kollár, Karen E. Smith, and Alessio Corti, Rational and nearly rational varieties, Cambridge Studies in Advanced Mathematics, vol. 92, Cambridge University Press, Cambridge, 2004. MR-2062787

[KX16] János Kollár and Chenyang Xu, The dual complex of Calabi-Yau pairs, Invent. Math. 205 (2016), no. 3, 527-557. MR-3539921

[MM86] Yoichi Miyaoka and Shigefumi Mori, A numerical criterion for uniruledness, Ann. of Math. (2) 124 (1986), no. 1, 65-69. MR-847952

[Mor82] Shigefumi Mori, Threefolds whose canonical bundles are not numerically effective, Ann. of Math. (2) 116 (1982), no. 1, 133-176. MR-0662120

[Oka09] Takuzo Okada, On the birational unboundedness of higher dimensional $\mathbb{Q}$-Fano varieties, Math. Ann. 345 (2009), no. 1, 195-212. MR-2520058

[PS09] Yu. G. Prokhorov and V. V. Shokurov, Towards the second main theorem on complements, J. Algebraic Geom. 18 (2009), no. 1, 151-199. MR-2448282

[Ryd06] Daniel Ryder, Classification of elliptic and K3 fibrations birational to some $\mathbb{Q}$-Fano 3-folds, J. Math. Sci. Univ. Tokyo 13 (2006), no. 1, 13-42. MR-2223680

[Sar80] V. G. Sarkisov, Birational automorphisms of conic bundles, Izv. Akad. Nauk SSSR Ser. Mat. 44 (1980), no. 4, 918-945, 974. MR-587343

[Sar82] _ On conic bundle structures, Izv. Akad. Nauk SSSR Ser. Mat. 46 (1982), no. 2, 371-408, 432. MR-651652 